\title{
Model Based Bolt Preload Monitoring Using Digital Image Correlation ${ }^{+}$
}

\author{
Carlos Jiménez-Peña ${ }^{1, *}$, Alberto Lavatelli ${ }^{2}$, Ruben Balcaen ${ }^{3, *}$, Barbara Rossi ${ }^{4}$ and \\ Dimitri Debruyne ${ }^{1}$ \\ 1 Department of Materials Engineering, KU Leuven, 9000 Ghent, Belgium; dimitri.debruyne@kuleuven.be \\ 2 Department of Mechanical Engineering, Politecnico di Milano, 20156 Milan, Italy; \\ alberto.lavatelli@polimi.it \\ 3 MatchID, 9031 Drongen, Belgium \\ 4 Department of Civil Engineering, KU Leuven, 3001 Leuven, Belgium; barbara.rossi@kuleuven.be \\ * Correspondence: carlos.jimenezpena@kuleuven.be (C.J.-P.); Ruben.Balcaen@matchid.eu (R.B.); \\ Tel.: +32-465-593-138 (C.J.-P.) \\ + Presented at the 18th International Conference on Experimental Mechanics (ICEM18), Brussels, Belgium, \\ 1-5 July 2018.
}

Published: 29 June 2018

\begin{abstract}
Bolt preload is one of the most significant parameters in bolted connections, which affects the static and fatigue performance of bolted connections. Traditional preload measuring methods give an estimation of the preload at the moment of tightening but cannot monitor preloading losses. In this investigation an initial proof of concept of a DIC based approach to relate bolt elongation to bolt preload is presented. Bolt elongation tests were performed to M16 bolts and a calibration method was proposed to obtain the bolt preload-elongation relationship for a given clamping length range.
\end{abstract}

Keywords: preload; DIC; bolted joints

\section{Introduction}

Bolt preload, also known as bolt pretension or clamping force, is the force applied to a bolt that compresses the joined components in a bolted connection and holds them together. This tension in the bolt is induced by tightening a nut on the thread of the bolt. The amount of preload will directly determine the design slip resistance in category $B$ and $C$ shear bolted connections, as indicated by the European standard for the design of joints (Eurocode 3, Part 1-8) [1]. It has been shown that the bolt preload can decrease the stress concentration at the bolted hole region and thus increase the tensile strength of the joint [2], and that it has an effect on the fatigue performance of the connection [3-6]. Consequently, the estimation of the bolt preload is of special interest to predict structural integrity and residual life-time in bolted assemblies.

The bolt tightening methods considered in the European Standard for Execution of steel structures and aluminum (EN 1090-2:2008) [7] evaluate the preload only at the moment of assembly while the loss of pretension is not monitored. Additionally, preload-measuring techniques based on torque values are greatly dependent on the lubrication condition and are prone to inaccurate measurements [8]. Multiple friction-independent preload monitoring methods already exist such as the use of load washers, strain gauges embedded in the bolt and even ultrasonic extensometry [9]. However, they require adding external elements to the bolted connection, proximity to electronic gear and/or expensive equipment, which limits their use to mainly research projects. Nonetheless each of the forementioned techniques has limitations when working in harsh or hardly accessible environments. An ideal preload monitoring technique would allow to perform contact-free 
measurements without modifying the bolted connection. Contact-less optical measuring techniques have already been employed to measure the bolt preload. In [10], the deformation of the washer during preload was related to the clamping force using Digital Image Correlation (DIC). In [11], a similar approach was used by measuring the out of plane deformation produced on the clamped surface using three-dimensional electronic speckle pattern interferometry (3D ESPI). However, these approaches are sensitive to the location of the extraction of the deformation field and are only applicable to specific bolted configurations or materials. This issue could be avoided if the analysis would be directly focusing on the direct measurement of axial actions due to tightening. Therefore, this paper presents a DIC based bolt elongation measurement method designed to obtain a reliable estimation of the clamping force. During the tightening process, a tensile force is applied to the bolt and, as a result, the bolt experiences an elongation. The magnitude of the elongation is directly related to the amount of preload in the bolt and can serve as alternative preload monitoring procedure.

This investigation contains an initial proof of concept of the DIC based approach. Firstly, a set of bolt elongation measurements were performed to confirm the suitability of DIC for accurately measuring the bolt elongation. Then the approach is applied to the analysis of static loading bolttightening under different clamping lengths. Finally, DIC is used to relate the bolt elongation to the bolt preload.

\section{Materials and Methods}

\subsection{Calibration Process}

The final objective of this study is to accurately measure the bolt elongation and relate it to the preload that has been applied to the bolt. It is well known that the bolt elongates linearly up to the proof load, which represents the usable preload range for standard fasteners [12]. Therefore, if a certain bolt preload-elongation curve is known, it would be possible to determine the bolt preload at any moment of its service life (after tightening, during service control, etc.). However, predicting the elongation of the bolt under a certain preload is not a simple task. When a bolt is loaded, the threaded portion of the bolt will tend to stretch more than the unthreaded one. Threads within the nut or within a tapped hole will also stretch less. The amount of engaged and unengaged thread stretch is directly affected by the fastener diameter. Calculations must be made using the exact lengths of both the unthreaded portion of the bolt and the remaining length of the unengaged threaded portion of the bolt as well as the diameter of the bolt. The complex contact condition among the thread peaks is also to be considered. Creating a reliable numerical model requires a great deal of experimental and numerical work, which will have to be modified for each bolt type.

An alternative calibration method is proposed in this study. The bolt can be considered as spring system in which the total elongation can be expressed as a function of the force acting on the spring and the length of the spring, as shown in Figure 1a. These variables can be interpreted in a bolt system as the bolt preload (spring force) and the clamp length (spring length). The clamp length is defined in EN 14399-4 [13] as the total thickness of the clamped parts, including the washers. A schematic view of the bolt system is shown in Figure 1b. If the bolt is subjected to its design preload force, the bolt elongation will ultimately depend on the clamp length only. In this way, by measuring the bolt elongation along a range of clamp lengths, the bolt elongation required to achieve the design preload can be obtained. As in the spring system, the elongation-to-clamping length relationship is expected to be linear.

The experimental setup is shown in Figure 1c. The clamp length is modified by adding steel plates within the bolt. The studied clamp length ranges from 36 to $78 \mathrm{~mm}$. The preload and the elongation are monitored during the tightening process, which is repeated twice for each clamp length to ensure repeatability. 


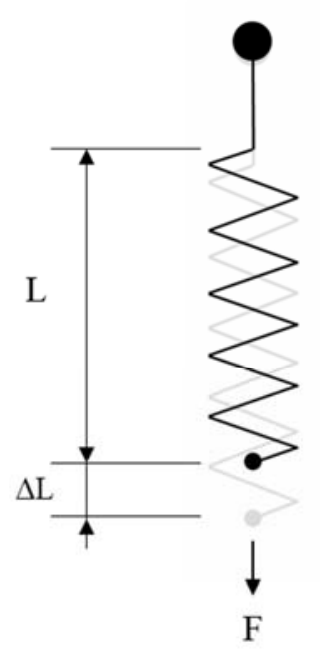

(a)

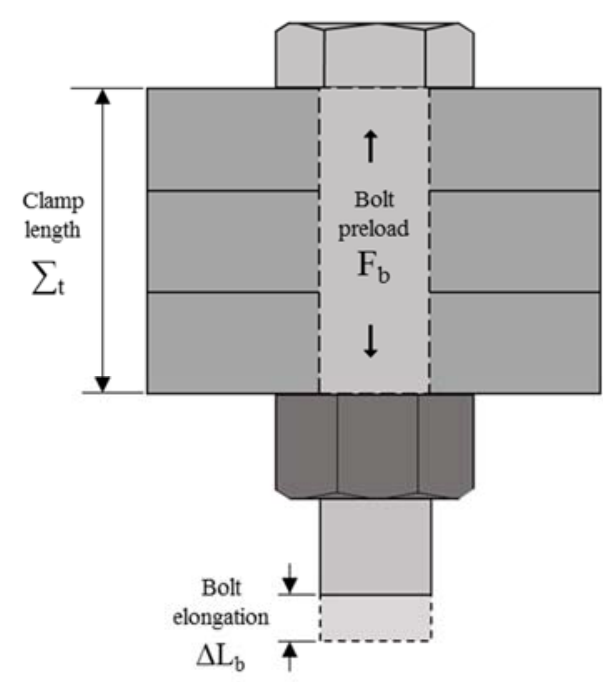

(b)

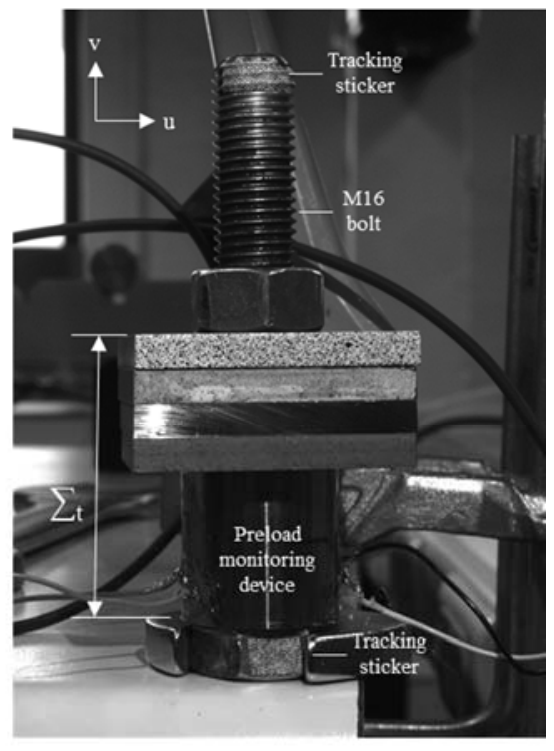

(c)

Figure 1. (a) Spring system; (b) Bolt elongation under a certain bolt preload; (c) Experimental setup.

\subsection{Bolt Geometry}

The bolt metric used in this investigation corresponds with the main bolt type employed in the European Research Project DuraMech [14], which are M16 bolts. This project deals with the fatigue behaviour of bolted connections in HSS. The aim is to directly apply the findings regarding the bolt preload evaluation to the bolted connections in the DuraMech project and therefore, the same bolt metric will be studied. This initial bolt elongation experiment was performed with full threaded ISO 4017 M16x100 8.8 bolts.

\subsection{Bolt Preload Measurement}

An instrumented washer with 1-LY41-6/350 strain gauges was fabricated to perform the bolt preload measurements. The strain gauges were attached to the outer sides of the load washer and aligned along the longitudinal direction of the washer. A schematic view of the load washer is shown in Figure 2. A compression test was performed to calibrate the strain-load relationship of the load washer. This calibration procedure was performed in a Zwick Z250 uniaxial tensile bench in displacement control at a speed of $0.1 \mathrm{~mm} / \mathrm{min}$.

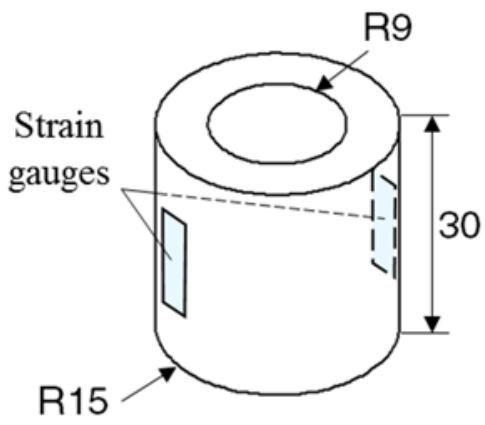

Figure 2. Load washer dimensions and location of the strain gauges [mm].

\subsection{Bolt Elongation Measurement with Digital Image Correlation}

DIC is an optical method for displacement and strain measurements that analyses and compares digital images acquired from the surface of a specimen [15]. Due to rapid new developments in high 
resolution digital cameras for static as well as dynamic applications and in computer technology, the number of applications of this measurement method increased. DIC technique has proven to be a flexible and useful tool for deformation analysis. It is used to monitor the displacement field, which is calculated based on the best correlation between the image of a deformed surface and a reference image of the un-deformed surface. This calculation is based on the Sum of Squared Differences (SSD) correlation criterion. The correlation process is only feasible if the surface of the specimen is covered by a random speckle pattern, which is in most cases applied with aerosol paint.

Herein, the digital images were taken by two Allied Vision Manta digital cameras, with a resolution of $2752 \times 2206$ pixels, mounted on a tripod positioned vertically in front of the bolted assembly. Both cameras had a lens with a focal length of $25 \mathrm{~mm}$. Two halogen lamps were used to improve lightning and contrast. In order to reduce any possible thermal interferences caused by the heat, the lamps were placed behind the cameras. The image noise was evaluated as $0.4 \%$ of the dynamic range. MatchID Stereo [16] is used to perform 3D DIC measurements. A summary of the DIC calculation settings is displayed in Table 1.

In order to measure the bolt elongation, the displacement fields of the bolt head and the bolt thread were extracted and the difference between the displacement magnitudes calculated using the following expression:

$$
\Delta l_{\text {bolt }}=\sqrt{\left(u_{\text {head }}-u_{\text {thread }}\right)^{2}+\left(v_{\text {head }}-v_{\text {thread }}\right)^{2}+\left(w_{\text {head }}-w_{\text {thread }}\right)^{2}}
$$

where $u, v$ and $w$ are the average displacement fields in each direction for the bolt head or the bolt thread, depending on the subscript.

Table 1. Parameters used in the DIC post-processing analysis.

\begin{tabular}{cc}
\hline Parameter & Value \\
\hline Correlation Coefficient & Zero-Normalised SSD \\
Interpolation order & Bicubic Spline \\
Transformation order & Affine \\
Subset size X step (pixels) & $21 \times 10$ \\
Noise handling & Gaussian $5 \times 5$ \\
History & Spatial \\
\hline
\end{tabular}

Contrarily to most research in which aerosol paint is used, in order to mark the surface of the bolt with a random pattern, stickers with a synthetically generated pattern were produced. They were attached to the bolt head and thread end to monitor the total bolt elongation with DIC (Figure 1c). Stickers are easier to apply and less sensitive to the deformation on the underneath bolt surface.

\section{Results and Discussion}

The bolt was preloaded twice for each clamping length and multiple elongation measurements were performed during each tightening process. The bolt preload-to-elongation relationship is linear as evidenced by the resulting measured bolt elongations, as shown in Figure 3a for the minimal and maximal clamp lengths. The scatter was also observed to be very low. A linear regression model was fitted to each clamp length case and the elongation at the design preload was calculated, see Figure 3a,b.

It can be noted that under the same preload, the bolt elongates more if a larger clamping length is used. The corresponding linear regression model can be defined as, for this type of 8.8 M16 bolts:

$$
\Delta L_{b}=0.0027 * \Sigma_{t}-0.0183
$$

This equation gives an estimation of the required bolt elongation that needs to be achieved to ensure that the design bolt preload is reached. Additionally, since the bolt preload-to-elongation relationship is linear, any subsequent loss of elongation during service allows to estimate the bolt loss of pretension. The bolt preload-elongation relationship can be easily derived for any clamping length from Equation (2) as: 


$$
F_{b}=\left(\frac{50}{0.0027 * \Sigma_{t}-0.0183}\right) \Delta L_{b}
$$

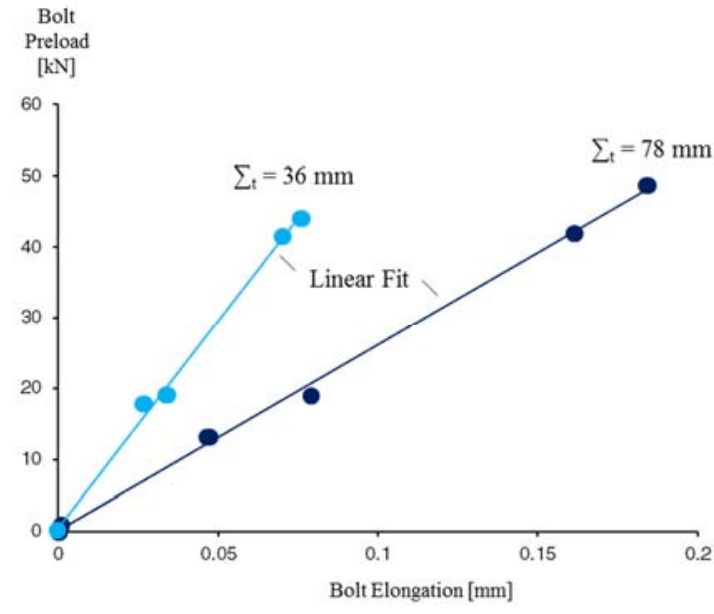

(a)

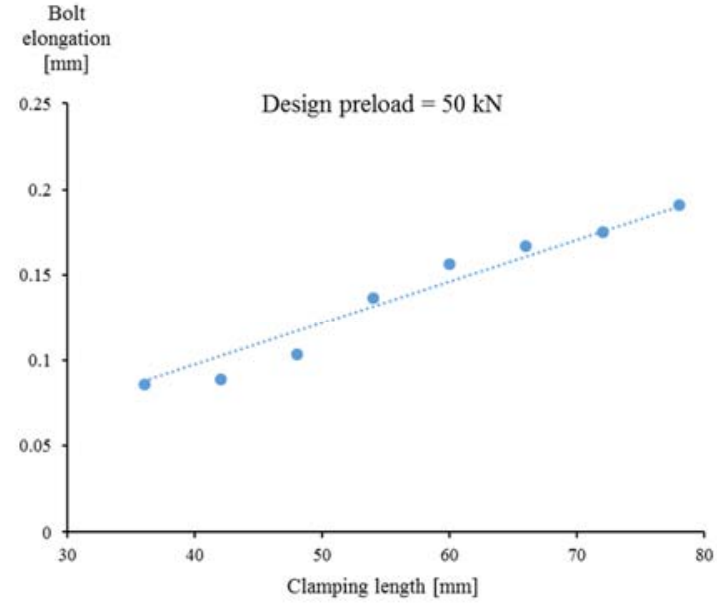

(b)

Figure 3. (a) Bolt preload-to-elongation curve for the minimal and maximal clamp lengths; (b) Bolt elongation under design preload for a range of clamping lengths.

\section{Conclusions}

This work presented a model-based contact-less bolt preload measurement technique based on DIC techniques. The ultimate goal is to demonstrate the possibility to measure the bolt preload only based on the total elongation of the system and its clamping length. The axial load was first evaluated based on the axial elongation via a linear elastic equivalent model. DIC was then used to provide accurate contact-less measurements of the axial elongation of a series of bolted connections. The analysis of the experimental data revealed a linear regression between the bolt elongation and preload for the range of studied clamping lengths and demonstrated the feasibility of the research question. At the same time, the experimental data highlight a set of limitations that need to be overcome to achieve the robustness required for a generic model intended for structural monitoring application. As a consequence, future researches will concentrate on how to enhance the repeatability of the results both in terms of calibration and of measurement. At the same time a deeper analysis of uncertainty will be carried out to highlight the strengths and pitfalls of the proposed method.

\section{References}

1. Standardisation ECf. EN 1993-1-8. Eurocode 3: Design of Steel Structures-Part 1-8: Design of Joints; CEN, Brussels, Belgium, 2005.

2. Yan, Y.; Wen, W.D.; Chang, F.K.; Shyprykevich, P. Experimental study on clamping effects on the tensile strength of composite plates with a bolt-filled hole. Compos. Part A Appl. Sci. Manuf. 1999, 30, 1215-1229.

3. Jiménez-Peña, C.; Talemi, R.H.; Rossi, B.; Debruyne, D. Investigations on the fretting fatigue failure mechanism of bolted joints in high strength steel subjected to different levels of pre-tension. Tribol. Int. 2017, 108, 128-140.

4. Benhamena, A.; Talha, A.; Benseddiq, N.; Amrouche, A.; Mesmacque, G.; Benguediab, M. Effect of clamping force on fretting fatigue behaviour of bolted assemblies: Case of couple steel-aluminium. Mater. Sci. Eng. A 2010, 527, 6413-6421.

5. Esmaeili, F.; Chakherlou, T.N.; Zehsaz, M. Investigation of bolt clamping force on the fatigue life of double lap simple bolted and hybrid (bolted/bonded) joints via experimental and numerical analysis. Eng. Fail. Anal. 2014, 45, 406-420.

6. Esmaeili, F.; Chakherlou, T.N.; Zehsaz, M.; Hasanifard, S. Investigating the effect of clamping force on the fatigue life of bolted plates using volumetric approach. J. Mech. Sci. Technol. 2013, 27, 3657-3664. 
7. EN B. 1090-2 (2008): Execution of Steel Structures and Aluminium Structures - Part 2: 2. Technical Requirements for Steel Structures; British Standards Institution: London, UK, 2008.

8. Ueno, T.; Yamaji, M.; Tsubaki, H.; Amasaka, K. Establishment of bolt tightening simulation system for automotive industry application of the highly reliable CAE model. Int. Bus. Econ. Res. J. 2009, 8, 57-67.

9. Koshti, A.M. Ultrasonic measurement and monitoring of loads in bolts used in structural joints. SPIE 2015, 9437, doi:10.1117/12.2083220.

10. Huang, Y.; Liu, L.; Yeung, T.; Hung, Y. Real-time monitoring of clamping force of a bolted joint by use of automatic digital image correlation. Opt. Laser Technol. 2009, 41, 408-414.

11. Nassar, S.A.; Meng, A. Optical monitoring of bolt tightening using 3D electronic speckle pattern interferometry. J. Press. Vessel Technol. 2007, 129, 89-95.

12. Bickford, J. An Introduction to the Design and Behavior of Bolted Joints, Revised and Expanded; CRC Press: Boca Raton, FL, USA, 1995.

13. European Committee for Standardisation. EN 14399-1:2005 High-Strength Structural Bolting Assemblies for Preloading-Part 4: System HV-Hexagon Bolt and Nut Assemblies; European Committee for Standardisation: Brussels, Belgium, 2006.

14. RFCS Project DuraMech. Towards Best Practice for Bolted Connections in High Strength Steels. Unpublished work.

15. Sutton, M.A.; Orteu, J.J.; Schreier, H. Image Correlation for Shape, Motion and Deformation Measurements: Basic Concepts, Theory and Applications; Springer Science \& Business Media: Berlin/Heidelberg, Germany, 2009.

16. MatchID Software. Available online: http://www.matchidmbc.be (accessed on 1 Februay 2018).

(C) 2018 by the authors. Licensee MDPI, Basel, Switzerland. This article is an open access article distributed under the terms and conditions of the Creative Commons Attribution (CC BY) license (http://creativecommons.org/licenses/by/4.0/). 